\title{
Classically conditioned heart rate and respiratory-motor activity in newborn and neonatal pygmy goats
}

\author{
ROBERT D. FITZGERALD, DAVID L. FRANCISCO, \\ JAMES METCALFE, and MARALEE S. LAWSON \\ Oregon Health Sciences University, Portland, Oregon
}

\begin{abstract}
Classical discrimination conditioning of heart rate (HR) and respiratory-motor (RM) activity was examined in 16 newborn (average age $=1$ day) and 8 neonatal (average age $=2$ weeks) pygmy goats over 4 days of conditioning involving an electric shock US and auditory CSs. On the 4 th day, one-half of the animals in each group received a low to moderate dose of ethanol to test the resistance of the CRs to drug effects. Discriminated conditioning of RM activity but not HR occurred in the newborn group, indicating an absence of close coupling between skeletal-motor activity and HR at this early age. While the newborn group failed to show recognizable HR reactions to the CSs during the 4 days of testing, it did show a long-latency bradycardia UR beginning at 2 days of age that may have been secondary to baroreceptor reflex actions. The neonatal group displayed conditioning of both HR and RM activity. The similar latencies of the two types of CRs during the CS suggested that they may have been related to each other. However, the fact that the RM CR developed a day before the HR CR, combined with the observation that ethanol modified the HR CR but not the RM CR, supports the view that there was considerable independence between the two types of CRs.
\end{abstract}

Learned changes in heart rate (HR) in adult subjects have appeared sometimes to be part of a more general cardiac-somatomotor response complex and other times to be relatively independent of motor activity. For example, experiments involving aversive classical conditioning have shown that HR CRs can occur in the absence of overt movement but that, given the presence of movement matching the direction of the HR CR, the magnitude of the HR change can be augmented (Cohen, 1969; Fitzgerald, 1966; G. K. Martin \& Fitzgerald, 1980; Obrist, Lawler, \& Gaebelein, 1974; Smith \& Stebbins, 1965). On the other hand, in situations in which overt motor activity occurs as part of the requirements of the experimental paradigm, changes in HR (whether conditioned or not is often difficult to determine) and motor responding have frequently been found to covary (Obrist, 1981).

Although relatively little is known concerning the behavioral relations between HR and skeletal-motor activity in young animals, some findings have been reported indicating that at certain ages nonspecific changes in both systems may not be linked to each

This research was supported by grants from the National Institute of Child Health and Human Development (HD-10034), the National Heart, Lung, and Blood Institute (HLO-7332), and the Oregon Heart Association. R.D.F. and D.L.F. are in the Department of Medical Psychology; J. M. and M.S.L. are in the Department of Medicine. Requests for reprints should be sent to Robert D. Fitzgerald, Department of Medical Psychology L470, Oregon Health Sciences University, 3181 S.W. Sam Jackson Park Road, Portland, OR 97201. other. Thus, during the first 2 weeks or so of life, spontaneous gross motor movements in rats were observed in the absence of corresponding changes in HR (Ashida, 1972; Hofer \& Reiser, 1969), suggesting that the two systems may develop at different rates. A specific classically conditioned HR CR was established in 3-4-day-old rats (L. T. Martin \& Alberts, 1982), but the question of possible motor involvement was not evaluated. Additional information on potential cardiacsomatomotor ties during early development could prove useful in our understanding of the biological significance of HR to adult behavior.

The purpose of the current experiment was to determine whether a composite respiratory-motor response accompanied a classically conditioned $\mathrm{HR}$ reaction in immature pygmy goats. Two groups of animals were examined; one group received conditioning as soon after birth as possible and the other group at approximately 2 weeks of age. Following 3 days of conditioning, half of the animals in each group received a low-to-moderate dose of ethanol prior to receiving additional conditioning trials. The administration of ethanol was used as a drug-challenge test (Spear, 1979) to evaluate the relative susceptibility to drug effects of the HR and respiratorymotor responses of the developing kids.

\section{METHOD}

\section{Subjects}

Twenty-four pygmy goats were used. Eight of the goats were 2 weeks old at the start of the study. The age of the remaining 16 goats varied between 16 and $32 \mathrm{~h}$, with the group average 
being $24 \mathrm{~h}$. The kids were housed with their mothers and other goats of various ages and numbers in $3 \times 3 \mathrm{~m}$ wire-mesh enclosures located within larger indoor rooms. In addition to nourishment gained from nursing, the kids had free access to water and dry food pellets.

\section{Apparatus}

The experimental chamber was a $61 \times 61 \times 80 \mathrm{~cm}$ Industrial Acoustics Corporation sound-attenuating room illuminated continuously by a 60 -W houselight mounted in the ceiling. To help mask extraneous auditory signals, white noise, averaging 75-dB SPL (re $20 \mu \mathrm{N} / \mathrm{m}^{2}$ measured at the approximate position of the animal's head), was continuously presented through a 10-cm speaker mounted on the back wall of the chamber. The kids were suspended above the floor of the chamber in a fullbody sling attached to the ceiling of the chamber.

The electrocardiogram (ECG) was recorded on a Grass Model 5 polygraph from two disk electrodes located opposite each other on the animal's chest. Heartbeats were counted in successive time intervals within each trial using an on-line computerized system (Fitzgerald, Vardaris, \& Teyler, 1968). The system consisted of a lever-type microswitch positioned on the frame of the polygraph so that the switch was activated by the $R$ wave of the QRS complex. Each switch closure triggered an electronic pulse shaper whose output was fed to an electronic counting network. At the end of preprogrammed time intervals within each trial, the cumulated heartbeat totals were encoded on paper tape using a Tally paper-tape perforator. Respiratorymotor activity was measured from the ECG electrodes using a Narco-Bio Systems Inc. impedance pneumograph (Model MKIV) that sensed changes in impedance between the ECG electrodes associated with breathing and gross body movements.

The CS was either an interrupted $2-\mathrm{kHz}(500-\mathrm{msec}$ on, $500-$ msec off) tone or a continuous $700-\mathrm{Hz}$ tone, presented at an average sound pressure level of $80 \mathrm{~dB}$ (re $20 \mu \mathrm{N} / \mathrm{m}^{2}$ measured in the same way as the white noise) through separate $10-\mathrm{cm}$ speakers located directly in front of the subjects. The US was a 1-sec, 10-Hz train of 150-V dc, .5-msec pulses of shock delivered through 2-cm-diam stainless steel electrodes taped to a shaved area on the upper thigh of each hindleg. The intensity of the US was checked periodically on an oscilloscope by measuring the voltage drop across a fixed resistor in series with the subject.

\section{Procedure}

The neonatal (2-week-old) and newborn (1-day-old) groups received identical treatment over 4 consecutive days. At the start of each day, all subjects were given $10 \mathrm{~min}$ to adapt to the test environment. On Day 1, eight trials with each CS were given in the absence of any USs to provide a measure of the original responses to the CSs. Discrimination conditioning began immediately thereafter and consisted of eight trials in which the $700-\mathrm{Hz}$ tone (CS+) was paired with the US at a CS-US interval of $10 \mathrm{sec}$ and of eight trials in which the $2-\mathrm{kHz}$ tone $(\mathrm{CS}-$ ) was presented alone. On Day 2 and Day 3, an additional 16 CS+ and 16 CStrials were administered. Prior to testing on Day 4 , one-half of the kids in each group received an intravenous infusion of $1.0 \mathrm{~g} / \mathrm{kg}$ of ethanol $(30 \%) \mathrm{v} / \mathrm{v})$ through a $21-\mathrm{ga}$ butterfly cannula inserted into an external jugular vein. The other halves of the groups were given a comparable volume of saline. The infusion was delivered slowly over a $50-60-\mathrm{min}$ period to prevent trauma to the vein and to minimize emotional reactions. After the 10-min adaptation period, an addiitonal $16 \mathrm{CS}+$ and $16 \mathrm{CS}-$ trials were given each group.

Heartbeats were measured in 14 consecutive time intervals on all trials. The first interval, located immediately prior to the CS, was $10 \mathrm{sec}$ long and provided a measure of baseline HR. The remaining 13 intervals were each 2 sec long and occurred during the CS (five intervals) and following the US (eight intervals). Because of artifacts, heartbeats were not tabulated during the shock or for $1 \mathrm{sec}$ following shock termination. The heartbeat totals were converted to beats per minute (bpm) and HR difference scores were calculated on each trial by subtracting the 10-sec pre-CS rate from each of the $2-\mathrm{sec}$ rates. Respiratory motor (RM) responses were hand scored from the polygraph records. A RM response was counted for a given trial when the amplitude of any pen excursion during the CS was more than twice as large as any pen movement during the 10 -sec pre-CS interval. Since it was not always possible to distinguish between $\mathrm{RM}$ responses that involved respiratory changes only and those that contained both respiratory and motor activity, both trial types were included in the analysis.

\section{RESULTS}

Pre-CS baseline HRs (CS+ and CS- baselines were combined after an appropriate analysis of variance failed to show that they were significantly different) of each group averaged over the CS-alone trials and over each of the 3 days of conditioning are shown in Figure 1. Baseline HR of the newborn group was consistently higher than that of the neonatal group. For the newborn group baseline HR increased over days, whereas for the neonatal group it decreased. A $2 \times 4$ (groups $X$ days) analysis of variance (ANOVA) provided a significant groups effect $[F(1,22)=9.38, p<.01]$ and a significant groups $X$ days interaction $[F(3,66)=2.94, p<.05]$. A follow-up Newman-Keuls test revealed that the two groups were significantly different from each other on Days 2 and 3 but not on Day 1 (including the CS-alone trials).

The RM and HR responses of each group to the CSs averaged over the final two CS-alone trials and over all trials on each day of conditioning are depicted in Fig. ures $2 \mathrm{~A}$ and $2 \mathrm{~B}$, respectively. Inspection of the figures reveals that both groups showed discrimination conditioning of RM activity but only the neonatal group dis-

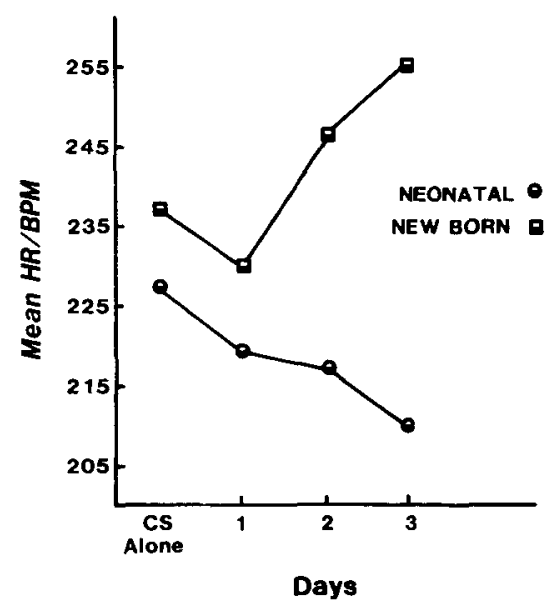

Figure 1. Mean baseline heart rate of each group averaged over the preconditioning CS-alone trials and over the conditioning trials each day. The CS-alone trials were given on the first half of Day 1. 

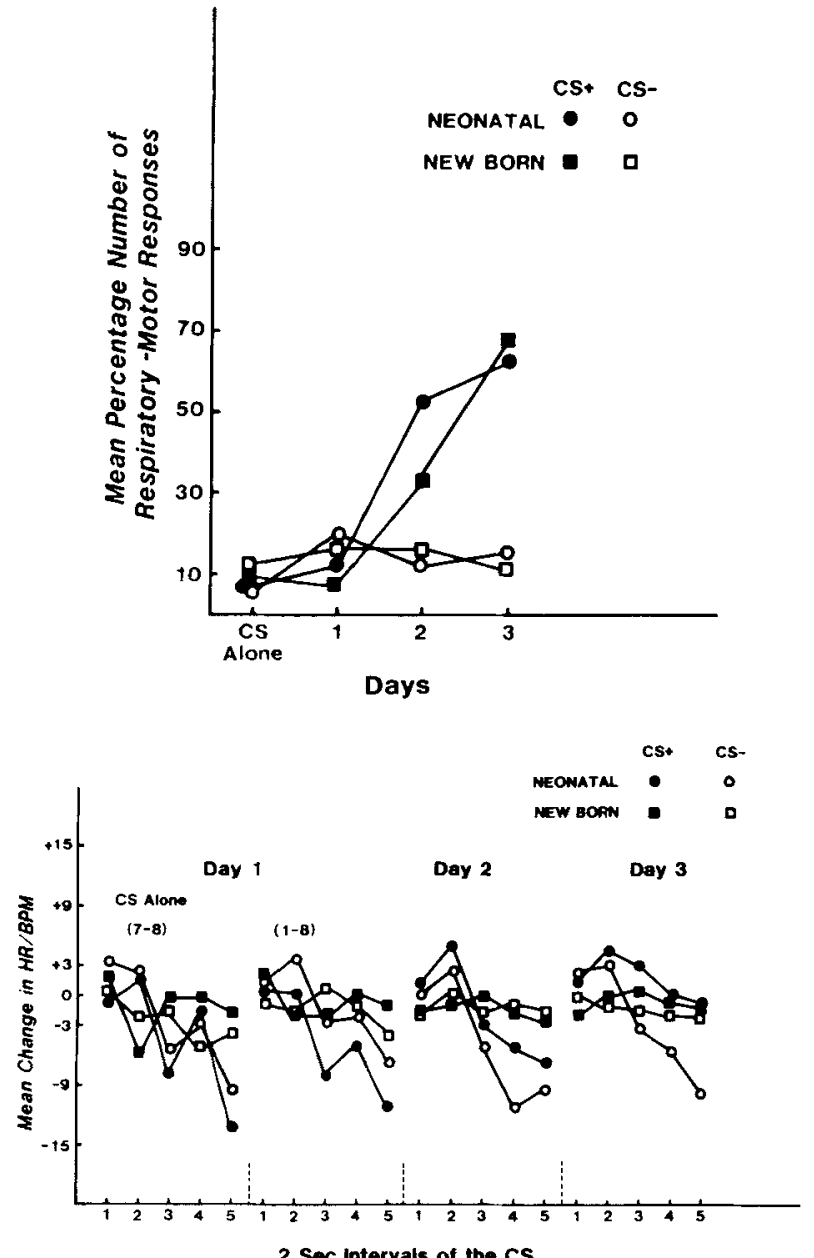

Figure 2. (A) Mean number of respiratory-motor responses of each group to the CSs on the preconditioning trials and on the conditioning trials each day. (B) Mean HR responses of each group in 2-sec intervals of CS+ and CS- on the last two preconditioning trials and on the conditioning trials each day.

played HR conditioning. The development of the RM CRs was evident on the second day of conditioning in both groups (RM data for two of the newborn animals was lost due to equipment failure). The RM CRs usually consisted of one or two deep inspirations, frequently combined with a brief burst of gross body movement. The mean latencies of the RM CRs of the groups were comparable and did not change significantly over days (overall mean $=2.85 \mathrm{sec}$ ). The neonatal HR CR (see Figure 2B) consisted of a gradual shift from a predominantly decelerative HR change to both CSs on the terminal CS-alone trials to an accelerative HR change to $\mathrm{CS}+$ on Day 3 at the same time that responding to CSremained mainly decelerative. Peak accelerative activity to CS+ occurred in the same measurement interval (number 2) as the average latency of the RM CR. The HR reactions of the newborn group were generally small-magnitude decelerations to both CSs on each day of conditioning.
A $2 \times 2 \times 4$ (groups $\times$ CS type $\times$ trials) ANOVA on RM activity gave a significant CS type effect $[F(1,20)=$ 20.82, $\mathrm{p}<.01]$ and a significant $\mathrm{CS}$ type $X$ trials interaction $[F(3,60)=40.41, p<.001]$, showing that $R M$ conditioning was reliable. Follow-up tests showed that there was a significant difference between CS+ and CSon Days 2 and 3 in the neonatal group, but only on Day 3 in the newbom group $(\mathrm{p}<.05)$. A $2 \times 2 \times 4 \times 5$ (groups $\times$ CS type $X$ trials $X$ measurement intervals) ANOVA on HR gave a significant interaction involving all four factors $[F(12,264)=2.34, p<.01]$. Separate three-way follow-up ANOVAs provided a significant CS type $X$ trials $X$ measurement intervals interaction $[F(12,84)=2.71, p<.01]$ for the neonatal group, indicating the presence of reliable HR conditioning. There were no significant outcomes in the HR analysis for the newborn group.

Postshock HR URs of the groups on each day are plotted in Figure 3. Here it may be seen that the UR of the neonatal group was a marked tachycardia on Day 1 and a biphasic tachycardia-bradycardia on Days 2 and 3. For the newborn group, there was virtually no tachycardia to shock on any day, but there was a substantial long-latency bradycardia on Days 2 and 3 that coincided with the fall in HR in the neonatal group. A $2 \times 3 \times 8$ (groups $\times$ days $\times$ measurement intervals) ANOVA provided a significant groups effect $[F(1,60)=$ $7.08, \mathrm{p}<.01$ ] and a significant groups $X$ days $X$ measurement intervals interaction $[F(14,308)=3.85$, $\mathrm{p}<.01]$, due mainly to the reduction in the magnitude of the tachycardia in the neonatal group over days.

\section{Day-4 Ethanol Challenge}

Following infusion, baseline HRs of the neonatal saline and ethanol subgroups were both elevated on Day 4 relative to Day 3 and were similar to the levels of the newborn subgroups (see Table 1). Resting HRs in the newborn subgroups on Day 4 were comparable to the high levels existing on Day 3. A $2 \times 2 \times 2$ (age group $X$ drug group $X$ days) ANOVA provided a signifi-

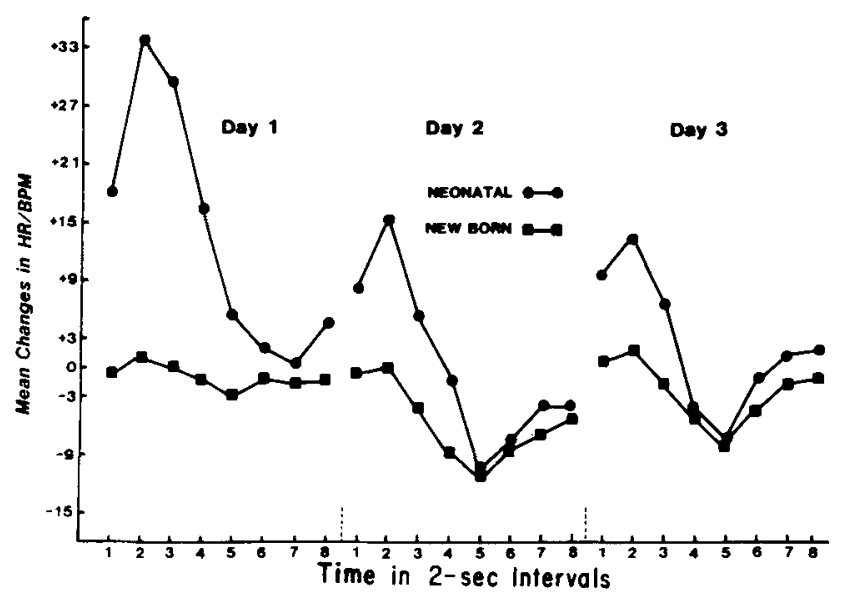

Figure 3. Mean unconditioned HR responses in 2-sec postshock intervals. 
Table 1

Pre-CS Baseline Heart Rate (bpm) of the Saline and Ethanol Subgroups on Days 3 and 4

\begin{tabular}{clcc}
\hline & & \multicolumn{2}{c}{ Heart Rate } \\
\cline { 3 - 4 } Group & Subgroup & Day 3 & Day 4 \\
\hline \multirow{2}{*}{ Newborn } & Saline & 255 & 247 \\
& Ethanol & 253 & 232 \\
\multirow{2}{*}{ Neonatal } & Saline & 216 & 250 \\
& Ethanol & 202 & 242 \\
\hline
\end{tabular}

cant age group $X$ days interaction $[F(1,20)=17.96$, $p<.001]$, showing that the differential change in the baseline HRs of the groups was reliable.

The HR responses of just the neonatal subgroups on Day 4 are shown in Figure 4 (one of the saline animals displayed a breath-holding bradycardia to shock on this day and was not included in the analysis of the HR

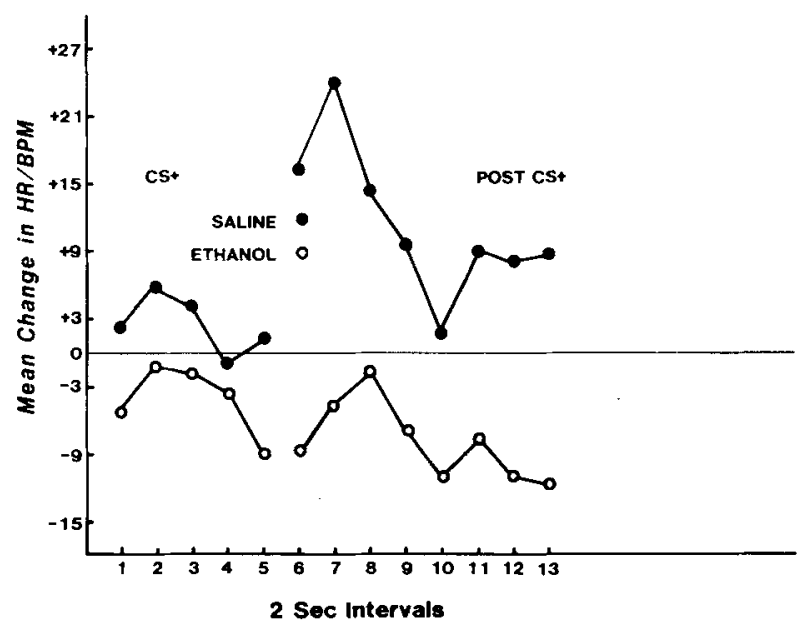

Figure 4. Mean HR responses of the ethanol and saline neonatal subgroups following infusion on Day 4.

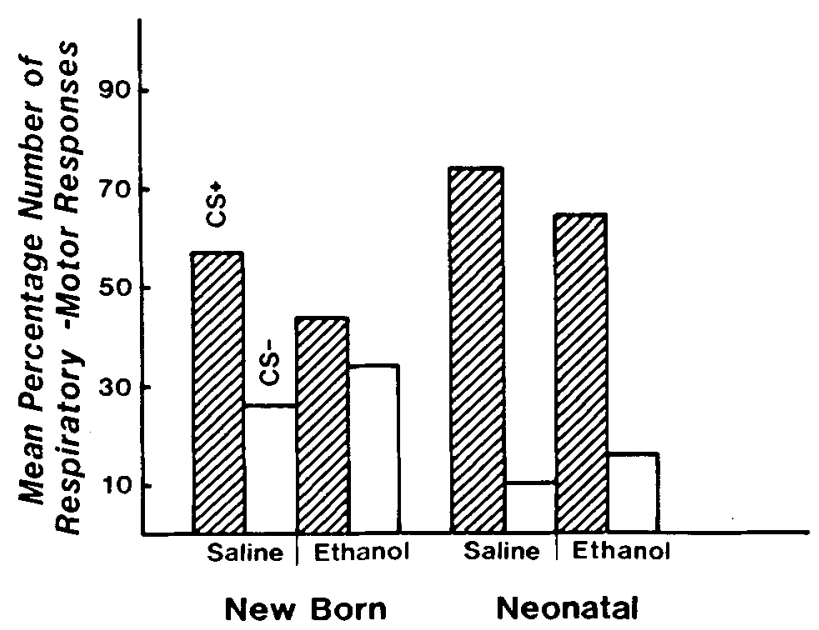

Figure 5. Mean number of respiratory-motor responses of the ethanol and saline newborn and neonatal groups to CS+ and CSfollowing infusion on Day 4.
URs). From this figure it may be seen that the neonatal ethanol group showed only decelerative HR changes to $\mathrm{CS}+$ and to shock as opposed to the accelerative reactions of the neonatal saline group. Although not shown, the HR response of the ethanol group to CS- was similar to that occurring to $\mathrm{CS}+$; that is, the discriminated HR CR was lost. The HR changes of both newborn groups to the CSs and the US continued to be small on Day 4, and there was no evidence of enhanced bradycardia under ethanol. Although a $2 \times 5$ (drug $\times$ measurement intervals) ANOVA on the neonatal subgroups failed to show that the ethanol suppression of HR acceleration to CS+ was significant, such an effect was obtained on early trials when trials (first half vs. second half) was added as a factor $[F(1,6)=15.40$, $\mathrm{p}<.01$ ] . A $2 \times 2$ (drug group $X$ measurement intervals) ANOVA on the shock responses gave a significant group effect $[F(1,5)=8.41, p<.05]$.

The Day 4 RM responses of the newborn and neonatal subgroups are shown in Figure 5. It may be seen that both newborn groups showed a decrement in RM discrimination performance in that responding decreased to $\mathrm{CS}+$ and increased to $\mathrm{CS}-$ relative to the neonatal groups. Neither neonatal group showed impaired RM performance. A $2 \times 2$ (age group $X$ drug group) ANOVA using the difference between CS+ and CS- as the raw data provided a significant age effect $[F(1,20)=8.76$, $\mathrm{p}<.01]$, showing that the loss of discrimination performance in the newborn groups was reliable.

\section{DISCUSSION}

The failure of the newborn group to show a discriminated HR CR can be attributed to the inability of their cardiovascular system to reflect conditioning, rather than to an inability to learn, inasmuch as this group did display a conditioned increase in RM activity comparable to that of the neonatal group. Except for a bradycardia response that appeared 6-10 sec following shock on the 2nd and 3rd day of conditioning, or approximately 2 days after birth, the newborn group showed an absence of identifiable HR responses to the CSs throughout the 4 days of conditioning. This low level of HR reactivity agrees with what has been reported for very young rats receiving auditory or visual stimulation (Ashida, 1972; Haroutunian \& Campbell, 1981), and suggests that, at least for some species, the processes mediating specific HR responses to certain types of environmental stimulation are not present at birth and require several days to develop post partum. The relatively long latency of the postshock bradycardia that was seen in the newborn group suggests that baroreceptor reflex pathways were functional shortly after birth. This response was similar to baroreceptormediated bradycardias that can be induced in sheep when blood pressure is elevated with a bolus injection of catecholamines (Floumoy, Camp, \& Krise, 1969).

The clear absence of a HR CR accompanying the well-developed RM CR that was seen in the newborn 
group demonstrates a substantial independence between the cardiac and motor systems lasting at least 4 days after birth. The neural pathways serving the RM CR did not elicit parallel changes in HR as would be expected if the two systems were coupled together centrally (Obrist, 1981). The capacity of the newborn group to form a RM CR within the first 3 days of birth is consistent with experiments (L. T. Martin \& Alberts, 1982; Rudy \& Cheatle, 1977) demonstrating successful conditioning in very young rats (2-3 days of age). In contrast to the present findings, one of these studies ( $L$. T. Martin \& Alberts, 1982) showed conditioning of HR. The presence of reliable HR conditioning in that study may have been encouraged by the use of an odor CS and either an illness-causing US or a temperature US, all of which are biologically very significant for infant rats. Whether a comparable combination of stimulus events would be effective for conditioning $H R$ in newborn pygmy goats cannot be determined from the current study.

In contrast to the newborn group, the neonatal group exhibited conditioning of both HR and RM activity. In general, the HR responses of the neonatal group to the CSs and the US were biphasic, consisting of tachycardia followed by bradycardia. Not unexpectedly, the US produced changes that were larger than those occurring to the CSs. The HR CR developed gradually and took the form of a tachycardia-only response to CS+, whereas the tachycardia-bradycardia reaction to CS- remained intact. Although the neonatal RM CR emerged prior to the HR CR (on Day 2, rather than Day 3), the tachycardia direction of the HR CR and the fact that the HR CR reached a peak at approximately the latency of the RM CR suggest that the two CRs may have been related to each other, perhaps as components of a conditioned "flight" reaction that some species exhibit to a CS danger signal (Cohen \& Obrist, 1975). Similar coupling of HR CRs and skeletal CRs have typically not been reported for adult animals, although surprisingly few attempts have been made, using appropriate controls, to determine the presence or absence of motor CRs during HR conditioning. In one study (G. K. Martin \& Fitzgerald, 1980) that showed such conditioning, restrained rats displayed a skeletal motor CR of an increase in EMG activity combined with a bradycardia $C R$, suggesting that what was conditioned was a behavioral state of "freezing."

More direct evidence that the HR and RM CRs of the neonatal group were not necessarily tied to each other was also seen. Following the administration of ethanol on Day 4, the HR CR of the neonatal ethanol group was transformed to a bradycardia (HR discrimination was lost), as was the tachycardia component of the UR. At the same time, discriminated RM responding of the neonatal ethanol group was not affected. Differential effects of ethanol on skeletal motor as opposed to cardiac responses have been reported in other experi- ments (Gantt, 1935), and the current findings extend those results to very young animals. In the present case, the profound modification in the direction of the neonatal HR response to $\mathrm{CS}+$ that occurred under ethanol in the absence of any visible change in the RM CR strongly suggests that the pathways serving the CRs were not completely overlapping. The loss of the neonatal tachycardias agrees with what has been observed in adult rats (Fitzgerald \& Stainbrook, 1978) and can be attributed to the suppressive effects of ethanol on cardiac control centers (Zsoter \& Seller, 1977).

Although the HR responses of the newborn kids were still too small to reveal a drug effect on Day 4, both newborn subgroups (saline and ethanol) did show a loss of discriminated RM responding. It appears likely that this impairment and the elevation in baseline HR that occurred in both neonatal subgroups on Day 4 were due to a general increase in emotionality following the infusions that were given on that day. Interference with discriminated performance under a high motivational level has been noted in certain situations (Yerkes \& Dodson, 1908). The fact that RM discrimination in the neonatal subgroups was not affected on Day 4 indicates that the older kids were better able to cope with the added stress brought on by infusion than were the very young kids.

Baseline HR of the newborn group was reliably higher than that of the neonatal group on Days 2 and 3 but not on Days 1 and 4. The failure of the groups to be different on Days 1 and 4 could have been due to an emotionally based elevation in the HR of the neonatal group. The occurrence of the higher resting HR in the newborn group is consistent with what has been found in other bovine animals such as sheep (Assali, Brinkman, Woods, Dandavino, \& Nuwayhid, 1978) and, based on findings with lambs (Assali et al., 1978), probably reflects a higher overall level of resting sympathetic tone in very young kids. The divergence between the newborn (a progressive increase) and the neonatal (a progressive decrease) baseline HRs across Days 2 and 3 provides further evidence of maturational differences between the groups and suggests that background sympathetic tone may have increased even further in the newborn animals. Such an increase could have represented an early developmental phase of sympathetic dominance in the control of baseline $H R$, as has been reported for rat pups (Hofer, 1974).

\section{REFERENCES}

Ashidn, S. (1972). Developmental changes in the basal and evoked heart rate in neonatal rats. Journal of Comparative and Physiological Psychology, 3, 368-374.

Assali, N. S., Brinkman, C. R., III, Woods, R., JR., DanDavino, A., \& Nuwayhin, B. (1978). Ontogenesis of the antonomic control of cardiovascular functions in the sheep. In L. D. Longo \& D. D. Reneau (Eds.), Fetal and newborn cardiovascular physiology (Vol. I). Development aspects (pp. 47-91). New York: Garland STM Press. 
Cohen, D. H. (1969). Development of a vertebrate experimental model for cellular neurophysiological studies of learning. Conditional Reflex, 4, 61-80.

Cohen, D. H., \& OBRIST, P. A. (1975). Interactions between behavior and the cardiovascular system. Circulation Research, 37, 693-704.

Fitzakrald, R. D. (1966). Some effects of partial reinforcement with shock on classically conditioned heart rate in dogs. American Journal of Psychology, 79, 242-249.

Fitzge Rald, R. D., \& Ste inb Rook, G. (1978). The influence of ethanol on learned and reflexive heart rate responses of rats during aversive classical conditioning. Journal of Studies on Alcohol, 38, 1916-1930.

Fitzgerald, R. D., Vardaris, R. M., \& Teyler, T. J. (1968). An on-line method for measuring heart rate in conditioning experiments. Psychophysiology, 4, 352-353.

Flournoy, R. W., Camp, B. J., \& Krise, G. M. (1969). Comparison in conscious goats of the effects of epinephrine, norepinephrine, and tryamine on blood pressure. Proceedings of the Society for Experimental Biology and Medicine, 181, 744-747.

GANTT, W. H. (1935). Effect of alcohol on cortical and subcortical activity measured by the conditioned reflex method. Bulletin of the Johns Hopkins Hospital, 56, 61-63.

Haroutunian, V., \& Campbell, B. A. (1981). Development and habituation of the heart rate orienting response to auditory and visual stimuli in the rat. Journal of Comparative and Physiological Psychology, 1, 166-174.

Hofe R, M. A. (1974). The role of early experience in the development of autonomic regulation. In L. V. DiCara (Ed.), Limbic and autonomic nervous systems research (pp. 195-221). New York: Plenum Press.

Hofer, M. A., \& Reiser, M. F. (1969). The development of cardiac rate regulation in preweanling rats. Psychosomatic Medicine, 31, 372-386.

Martin, G. K., \& Fitzon rald, R. D. (1980). Heart rate and somatomotor activity in rats during signalled escape and yoked classical conditioning. Physiology \& Behavior, 25, 519-526.

Martin, L. T., \& Alberts, J. R. (1982). Associative learning in neonatal rats revealed by cardiac response patterns. Journal of Comparative and Physiological Psychology, 4, 668-675.

Oвnist, P. A. (1981). Cardiovascular psychophysiology. A perspective. New York: Plenum Press.

Obrist, P. A., Lawler, J. E., \& Gaebelein, C. J. (1974). A psychological perspective on the cardiovascular system. In L. V. DiCara (Ed.), Limbic and autonomic nervous systems research (pp. 311-331). New York: Plenum Press.

Rudy, J. W., \& ChentLe, M. D. (1977). Odor aversion learning in neonatal rats. Science, 198, 845-846.

Smith, O., \& Stebins, W. C. (1965). Conditioned blood flow and heart rate in monkeys. Journal of Comparative and Physiological Psychology, 59, 432-436.

SPEAR, L. P. (1979). The use of psychopharmacological procedures to analyse the ontogeny of learning and retention: Issues and concerns. In N. E. Spear \& B. A. Campbell (Eds.), Ontogeny of learning and memory (pp. 125-155). Hillsdale, NJ: Erlbaum.

Yerkes, R. M., \& Dodson, J. D. (1908). The relation of strength of stimulus to rapidity of habit-formation. Journal of Comparative Neurology and Psychology, 18, 459-482.

Zsoter, T. T., \& Selle R, E. M. (1977). Effect of alcohol on cardiovascular reflexes. Journal of Studies on Alcohol, 38, 519-526.

(Manuscript received September 19, 1983; revision accepted for publication March 12, 1984.) 